\title{
Full-Frontal Morality: The Naked Truth about Gender
}

\author{
TALIA MAE BETTCHER
}

This paper examines Harold Garfinkel's notion of the natural attitude about sex and his claim that it is fundamentally moral in nature. The author looks beneath the natural attitude in order to explain its peculiar resilience and oppressive force. There she reveals a moral order grounded in the dichotomously sexed bodies so constituted through boundaries governing privacy and decency. In particular, naked bodies are sex-differentiated within a system of genital representation through gender presentation-a system that helps constitute the very boundaries between the public and private.

Since Harold Garfinkel's landmark "Passing and the Managed Achievement of Sex Status in an 'Intersexed' Person, Part 1" (in Garfinkel 1967), the notion of a natural attitude about sex has been deployed to advantage in multiple works in gender studies. The notion plays an important role in Suzanne Kessler and W. McKenna's Gender: An Ethnomethodological Approach (Kessler and McKenna 1978), Kate Bornstein's revolutionary Gender Outlaw: On Men, Women, and the Rest of Us (Bornstein 1994), and C. Jacob Hale's pioneering scholarship in trans studies (Hale 1996; 1997; 1998a; 1998b).

Garfinkel's appeal to a natural attitude draws from Edmund Husserl's phenomenology. For Husserl, there exists a pre-theoretical, common-sense view according to which the natural world exists independently of our minds (Husserl 1983 §30). The ethnomethodologist, like Husserl's phenomenologist, proceeds by "bracketing" this attitude. She examines concrete social interactions as phenomena with the guiding question: "What are the methodological ways by which members of a group produce, in each particular situation, this sense of external, constant, objective facts which have their own independent 
existences, not contingent on any concrete interaction?" (Kessler and McKenna 1978, 5).

Garfinkel outlines "axioms" of the natural attitude about sex, which can be schematized as follows. First, there is a commitment to natural sexual dichotomy: exactly two (mutually exclusive) sexes exist, every human being is "naturally" one or the other, and exceptions to this division may be dismissed as "unnatural." Second, there is a commitment to the natural invariance of sex: a human being can never change sex, and any sex transfers are merely ceremonial. Finally, there is an almost exclusive appeal to genitalia: genitals essentially determine sex membership (Garfinkel 1967).

Several of these beliefs are plainly false. Given the multiple features of sex determination (karyotype, gonadal structure, hormone levels, genital structure, and so on), it's hardly clear that genitalia are definitive. Moreover, in some people these features come apart, making it doubtful everyone is naturally one or the other. Yet these beliefs are significantly bound to everyday practice, so "normals" distrust theory and science and dismiss such considerations out of hand. In light of the falsity of the beliefs, it's unsurprising that the natural attitude about sex isn't universal. On the contrary, Kessler and McKenna adduce evidence of variable cultural conceptions of gender (including reference to "berdache") that don't adhere to the "axioms" (Kessler and McKenna 1978, 21-41). If the natural attitude is a "common sense" about the world, it's not one shared by everyone.

Implicated in the relation between the natural attitude and daily practice is the fact that the attitude is fundamentally moral. Garfinkel speaks of the normal's view of the "incompetent, criminal, sick, and sinful" who stand in "moral" contrast with "natural males and females" (Garfinkel 1967, 122). He says "this naturalness carries along with it, as a constituent part of its meaning, the sense of its being right and correct, i.e., morally proper that it be that way" (123). Moreover, he talks about penises and vaginas seen by the normal as "legitimate possessions" to which males and females respectively have moral entitlements (127). In effect, the natural attitude about the metaphysics of sex is also a view about a moral order.

This notion is useful in understanding a kind of transphobia thoroughly imbued with both moral and metaphysical considerations. It isn't uncommon for a trans person to be represented as "really a so and so, disguised as a such and such." A trans woman, for example, may be represented as engaging in a kind of "sexual deception." Ironically, although such "deceivers" are held accountable for misrepresentation, they're subjected to an often unacknowledged sexual violence in the form of genital verification to determine sex. Thus, while trans people may find their identities overtly invalidated through moral accusation, they may also be subjected to covert sexual violence within a framework juxtaposing appearance and reality. 
My aim in this paper is to look beneath the natural attitude in order to explain its peculiar resilience and oppressive force. There we will find a moral order based in dichotomously sexed bodies constituted through boundaries governing privacy and decency. In particular, naked bodies are sex-differentiated within a system of genital representation through gender presentation-a system that helps constitute the very line between public and private.

In this account, personhood is defined through such boundaries. Although the boundaries are conventional, they're grounded in the legitimate demands of intimacy. This means violations of the person can constitute veritable moral wrongs despite the fact that they're instituted in (hetero)sexist and transphobic ways. Resistance to the moral order involves far more than merely a change in belief. It involves the renegotiation of the moral boundaries involved in the constitution of the sexed body and personhood itself.

To be clear, I take this system of interpersonal boundaries to be both culturally and historically relative, like the natural attitude. I'll speak, quite roughly, of Eurocentered culture to refer to dominant cultural forms of life situated in the U.S./European-centered context of the present day. This doesn't mean other cultures don't have boundary systems that overlap with the one I describe (and that consequently sustain transphobia). However, I'm interested in describing a system of intimacy with a specific history of colonialism. One of my goals is to elucidate some of the ways this particular system institutes, as part of the moral fabric, a multiply oppressive (transphobic, sexist, homophobic, racist, ablest) construct of intimate personhood. In doing so, I recognize multiple subcultures, even within the United States, for example, with alternative, resistant systems.

Let me also disclose that I write this paper as a white transgender woman situated in Los Angeles within several intersecting trans, lesbian, and other resistant subcultures. It's in part because I'm able to see the dominant system from the vantage points of resistant places that I'm able to outline it at all. At any rate, my vision of the system is informed and limited by these several cultural vantage points. Since the project is geared primarily toward understanding transphobia, there will inevitably be distortions caused by that particular focus (as opposed to the inevitable distortions caused by another kind of focus). Suffice it to say I offer this account as a socially located contribution intended to be situated within a larger dialogue.

\section{The Social Construction of NaKedness}

One way a body can be viewed as socially constructed concerns its constitution as naked. My view is not merely that a naked human body can have different cultural meanings assigned to its nakedness. Rather, my view is that nakedness itself is a specific construct. Infants aren't born wearing clothing; they're 
covered up with culturally designed artifacts by other human beings. That's a social practice. However, naked is defined relative to clothed. Without the concept of being clothed, there could be no concept of nakedness. Similarly, without the social possibility of being clothed, there could be no corresponding social possibility of being naked.

In a culture lacking the concept of nakedness and the practice of body concealment, one might very well describe the members as "naked." However this conception of nakedness stands in contrast with the way members of a culture can be naked or not precisely because a social practice exists of wearing clothing; here, nakedness is a well-defined (socially constituted) modality of selfpresentation, contrasting with other modalities of self-presentation.

Nor should one believe that the social practice of wearing clothing has any "natural" grounding. In addition to the existence of nudist subculture, there have been many cultures in which bodily concealment was not a salient social practice, for example, among aboriginal peoples (see Barcan 2004, 138, 15659). To be sure, the phenomenon of bodily adornment seems fairly ubiquitous (see Nagel 2002, 16), and how one defines nakedness is a tricky business (see Barcan 2004, 16, 284). Yet the fact remains that such bodily concealment isn't universal. Aboriginal scholar Irene Watson writes, for example:

There are no words that I have come across in our indigenous languages to describe nakedness. Prior to the colonists' invasion of our territories there was no reflection of our nakedness. The reflection of nakedness came with the other, the clothed colonising peoples. Now there are few who physically walk the land naked. (Watson 1998, 2)

In Eurocentered culture, the social possibilities of being naked and clothed are bound with issues of morality, privacy, decency, and violation. ${ }^{2}$ To help describe this, I distinguish between the morally and materially private. ${ }^{3}$ The former involves a claim about the moral status of a body part, the latter a claim about whether a body part is in fact private (in the sense of being concealed from sight). In this latter sense, someone who "streaks" across a field has parts that aren't materially private. Regardless, they're morally private insofar as exposure elicits moral concern.

In Eurocentered culture, the moral privacy of body parts is partially constitutive of the social phenomenon of nakedness. In particular, the moral privacy of these parts undergirds the social practice of concealing them; it does so by referencing the moral consequences of nakedness. Because of the moral consequences of displaying one's privates, one conceals them in public. Not only does the social possibility of being clothed yield the social possibility of nakedness, the very practice of wearing clothes is motivated by reference to the possibility of nakedness as a moral phenomenon: we cannot separate the social 
phenomenon of nakedness from the status of certain body parts as morally private. That is, in Eurocentered culture, nakedness, insofar as it connects meaningfully to social practices, is thoroughly invested with moral meaning.

\section{Boundaries And People}

In saying body parts are "morally private" I mean they're morally bounded with respect to the sensory access of another. I take these moral boundaries to have two sides: one regulating the subject of perceptual access, one regulating the object. In Eurocentered culture, one way to transgress a boundary is to look at a person's privates without her consent. Here, privacy violation and a violation of the object occur. However, it's also appropriate to speak of decency violations and violations of the subject. This is typified when an individual inappropriately shares personal information. Another might say, "TMI!" (that's "Too Much Information!"). The boundary is transgressed by providing information that the subject has the right not to know. ${ }^{4}$ Similarly, one might show too much of one's body to another. Indeed, if a person deliberately exposes himself to another, this counts as a violation in Eurocentered culture. It's why, in part, laws against public indecency exist.

So even though these boundaries inhere in individuals' bodies, they also function as interpersonal boundaries that draw moral lines between people. That is, these boundaries are shared boundaries; they don't merely bound us, they bound others. Moreover, in most traditional face-to-face human interactions both parties are perceiving objects and subjects. This means two doublesided boundaries are in play.

This affords us a way to understand why privacy and decency violations can be violations of the person herself. We can allow a sense in which a person is delimited by her interpersonal boundaries, just as countries are delimited by the boundaries that separate them. In this sense, a person is defined by her interpersonal boundaries: to violate a person is to transgress a boundary that delimits her.

Caution is required in saying this. I don't wish to advance a general account of personhood. Philosophers use person in different ways, and much depends on how they use this word. In this paper, I'm interested in an understanding of person as mediated through our various relations to people. People we actually encounter in life are experienced through various interpersonal interactions that admit of varying degrees of intimacy. I want to avoid abstracting a notion of person from such relations. Rather, the sense of person that concerns me is brought out in one of the Oxford English Dictionary's definitions of intimate: "proceeding from, concerning, or affecting one's inmost self; closely personal." My claim is that what I call the intimate person is delimited in terms of interpersonal boundaries. 
In one sense, intimate can apply to particular features of an individual person; it may be used as a synonym for private or personal. In another, it can pertain to relations between people. According to another OED definition, intimate means "close in acquaintance or association; closely connected by friendship or personal knowledge; characterized by familiarity (with a person or thing); very familiar." Intimacy is defined as "the state of being personally intimate; intimate friendship or acquaintance; familiar intercourse; close familiarity." The key notions here are closeness and familiarity. ${ }^{6}$

The notion of closeness suggests a spatial metaphor; we might think of a kind of interpersonal distance or proximity. One obvious way of illuminating this is in terms of epistemological familiarity. Emotional closeness involves sharing biographical information, with a significant emphasis on one's affective attitudes. Physical closeness involves an increased sensory access to one another (visual access, touching, or at least an increased literal physical closeness). In general, intimacy can be understood in terms of mutual informational and/or sensory access to one another.

This notion of closeness suggests intimacy between people is a matter of degree. Although this is somewhat true, we shouldn't ignore the polarity between notions of interpersonal distance and closeness. Intimacy marks a divide between types of interpersonal relations. Intimacy is suggestive of a kind of boundary-traversing familiarity (not just familiar, very familiar). This bounded quality is required for intimacy. Without it, we'd merely have degrees of familiarity. However, the bounded nature of intimacy is made possible precisely by the interpersonal boundaries we've been discussing. ${ }^{7}$ Certain parts of the body are subject to boundaries on sensory access. Actual spatial distance between people is subject to a boundary. Biographical information, particularly attitudinal in nature, is subject to boundaries. Things are bounded as "personal" precisely because they're constitutively allocated to intimate relations. That is, they're required to constitute intimate relations as intimate. Interpersonal boundaries separate individuals with regard to sensory and informational access. Such boundaries exist so they may be, under appropriate conditions, mutually traversed. In effect, intimacy is made possible by interpersonal boundaries that delimit people. These people are thereby constituted as fit candidates for intimacy - people who are capable of close, moderate, and distant relations with one another. ${ }^{8}$ As a condition of this ability, they're constituted as susceptible to being violated by and to violating others. In effect, intimate personhood is profoundly moral in nature. ${ }^{9}$

I see the intimate body as playing a foundational role in the constitution of the intimate person. Admittedly, the intimate person is determined by boundaries besides those that govern sensory access to bodies. In particular, boundaries exist governing the exchange of biographical information (particularly information about affective state). However, it still seems that the moral 
privacy of the body is in some ways basic. Boundaries of the intimate body regulate sensory access rather than discourse; how we literally experience each other seems more fundamental than what we say about each other. Indeed, much information that is subject to boundaries precisely concerns the bounded body or its activities.

This conception of the intimate body and person is useful in illuminating how a moral order can be conventional and yet binding. One example is precisely the relativity of nakedness, which principally concerns a boundary on visual access. That a culture should possess such visual boundaries at all in addition to ones governing interpersonal space and tactile bodily contact is conventional. Yet the requirement that there be interpersonal boundaries derives normative force from the positive value of intimacy. A transgression of the boundary can constitute a genuine violation - a genuine moral wrongsince it involves the violation of the intimate person herself. Even though the exposure of genitals in public is merely a conventional transgression, the conventionality does nothing to undermine such exposure as a veritable sexual violation.

Furthermore, nakedness as a social construct is a good example of the way different kinds of bodies are privileged as normative. In Eurocentered culture, nakedness plays a significant role in the culture's particular system of intimacy. Insofar as nakedness primarily concerns vision as a modality of bounded access, sighted individuals are presupposed. This means that blind individuals can be positioned eccentrically (that is, marginalized and erased) with regard to the very institution of personhood as constituted within Eurocentered culture. That is, moral resources are differentially distributed on the basis of normatively selected bodies.

\section{Moral StRUCTURES AND THE BODY}

The human body possesses a moral structure determined by interpersonal boundaries. The most obvious example is the fact that in Eurocentered culture, although certain parts (genitalia) have boundaries on visual access, other parts (face and hands) do not. Because the face and hands are characterized by a lack of boundary on visual access, their lack of material concealment can't contribute to a body's nakedness. Of course, this is clearly a culturally relative moral structure - as is born out, for example, by the use of the burqa in some Muslim contexts. ${ }^{10}$ This suggests ways religion can be implicated within various systems of intimacy as well as how a dominant, Western, Eurocentered system of intimacy may be shaped by an internally invisible Christian normativity.

Although other parts are morally bounded, they aren't bounded to the same degree. Although buttocks are culturally coded as private in Eurocentered culture, they aren't as private as genitalia. Additionally, parts that aren't morally 
private are nonetheless bounded in other ways - such as through sheer "social inappropriateness": one can be almost naked on the beach, whereas one could never wear a swimsuit to church. All of this is subject to regulations of decorum. This kind of regulation is of a piece with the regulation of bodily access through sensory modalities.

The body, then, has a moral structure determined by what's bounded, how it's bounded, to what degree it's bounded, and why it's bounded. In Eurocentric culture, the bounding of genitalia occurs within the context of a larger structure of boundaries. Yet, although genitalia are "outstripped" by a larger structure, they also play a central role. First, a vague order is reflected in the overall moral structure of the body with regard to the movement from being clothed to being naked. Although merely exposed genitalia do not constitute nakedness as such, this exposure constitutes the "last stage" thereof or the completion of total nakedness. ${ }^{11}$ Second, genitalia are morally decisive with respect to nakedness. Insofar as nakedness is a moral concept, questions about whether a person is naked are answered in terms of genitalia. In the Eurocentered system, genitalia are fundamental to the moral structure of the intimate body. To capture this concept, I say that genitalia "morally complete" a body.

The structure of an intimate body, in Eurocentered culture, is fundamentally gendered. Boundaries on genitalia are linked to a particular kind of physical/ sexual intimacy, namely coitus. In effect, one main reason genitalia are morally delimited at all is to allocate them constitutively to a kind of physical intimacy. Within a (hetero)sexist context, coitus occupies a decisive location within the multiple possibilities of sexual interaction. It's that toward which all other sexual activities tend. This is reflected in the heterosexist and transphobic way in which laws against acts of sexual violence may define rape, the most culturally recognized and canonical form of boundary violation, strictly in terms of coitus (using categories such as sodomy to capture other forms of sexual violence). ${ }^{12}$

Not only are bodies divided according to two types of genitalia, these divided bodies are subject to differential structures. One example of this is the fact that a female structure involves two-tiered nakedness: the female chest is subject to a boundary that the male chest is not. This provides for the possibility of female "toplessness" and "bottomlessness" and hence affords nakedness twice over.

This difference in structure is reflected in the way the boundaries work. Rape is canonically conceptualized "male-to-female." One reason for this concerns the constitution of male genitalia as violating and female genitalia as violated. This is reflected in cultural ideology that sex with men harms or "dirties" women —an ideology that continues to have consequences today. Although women are no longer necessarily valued for purity in Eurocentered culture, it remains true, in certain cultural contexts, that a woman who has "too much sex" or sex "with the wrong people" or "in the wrong 
circumstances" will be subject to social sanction in a way that a man will not. I believe this is because sexual intercourse continues to be viewed as a boundary violation (and a violation of the woman herself), regardless of her consent.

I see such boundaries as prior to consent and motivational with regard to it. Hence, in order to avoid a pre-consensual boundary violation, a woman may avoid certain activities. Such pre-consensual boundaries constitutively allocate a woman's sexuality to particular elevated relations of intimacy that can then "clean" the sex of its capacity for boundary violation. The fact that marriage is no longer required as the elevated relation of intimacy doesn't mean such an asymmetrical system doesn't, to some extent, remain in play.

This type of asymmetry is also manifested in privacy/decency violations. It may be that a man viewing the naked body of a woman, in the absence of legitimizing conditions, constitutes a pre-consensual violation of her privacy (and hence, of her). By contrast, it doesn't seem to be the case that a woman viewing the naked body of man, in the absence of legitimizing conditions, constitutes a pre-consensual violation of his privacy. On the contrary, exposure of his body constitutes a pre-consensual decency violation (and hence a violation of her). Therefore, an asymmetry exists in the motivation for concealment in the presence of the "opposite sex": a female will cover up to protect her privacy, whereas a male will cover up to prevent his body from offending through indecency. ${ }^{13}$

Although this claim is no doubt overly simplified, it helps explain the underlying gender segregation of public restrooms, showers, and changing rooms, as well as segregation in congregate housing and strip-search requirements (which include requiring that police officers be the same sex as the detainee). One reason for segregating public showers, for example, is that pre-consensual boundaries restrict visual access between males and females. Presumably, this is because such intimate visual access is constitutively allocated to coitus and the path that leads there.

For evidence of the above-mentioned asymmetry, consider Paul Scott, a Michigan state representative and a failed GOP candidate for Secretary of State. In his bid for the candidacy he identified one of his top four priorities as ensuring that "transgender individuals will not be allowed to change the sex on their driver's license in any circumstance" (Heywood 2010). He explains the mandate was about "preventing people who are males genetically from dressing as a woman and going into female bathrooms." Gary Glenn, leader of the American Family Association of Michigan, elaborates: "I think there are all kinds of implications to the privacy rights of women and children if biological males are given access to health clubs, showers, locker rooms, and changing areas" (Harger 2010). The worry is that "biological males" will violate the privacy of females simply by seeing them, not the other way around. This formulation is no accident. 
Here we find more ways a conventional order can be both morally binding and subject to critique. The constitution of privacy/decency boundaries yields a situation in which a woman can be violated either through seeing or being seen by a man. The sexist nature of the order doesn't change the fact that violations of these boundaries constitute veritable moral wrongs. In this way, the moral order possesses an ambiguous character: On the one hand, it's oppressive. On the other hand, it's real. This ambiguity is made possible by the fact that relations of intimacy require interpersonal boundaries to constitute intimate persons, but leave entirely open the way those boundaries are drawn.

\section{The Meaning of Gender Presentation}

In Eurocentered culture, a person's genital status is socially constituted as an important moral fact. Since coitus is recognized as the completion of sexual intimacy, it will seem important to know in advance "who has what" in aiming for it, avoiding it, and regulating it (through various forms of segregation). Yet, genitals are generally materially private.

Perhaps enough differences in morphological features (such as height, muscle mass and fat distribution, bone width, Adam's apple, voice pitch, etc.) can serve as evidence for hypotheses about genitalia. To me, such a suggestion significantly underestimates the degree of cultural work needed to ensure that we can tell the sexes apart. Moreover, since cultural artifacts conceal the private body, cultural work is already afoot. That this work doesn't de-emphasize differences is necessitated by the fact that the morally private body is dichotomized in socially relevant ways.

To better understand this cultural work, I take presentation to be a presentation of one's embodied self. It includes attire, grooming, adornment, bodily gesture, posture, manner of speech, and socially interactive style. Given that such presentation is gender-differentiated, I speak of gender presentation. Obviously, much gender presentation is conventional and arbitrary in nature.

Consequently, gender presentation bears some analogy to human languages (in which arbitrary and culturally variable symbols are assigned to objects). Moreover, behavior in accord with a norm may provide evidence about genital status (just like certain morphological features do). However, the fact that the behavior provides evidence of genital status does not establish the behavior as literally communicative and representational. The following argument does.

In light of the social salience of genital status in Eurocentered culture, it seems like good fortune that normed behavior should provide evidence for it. Indeed, not only is the status socially salient, it has been socially privatized. That a need to know exists in the first place is a consequence of social facts. It's a remarkable coincidence, then, that socially regulated gender presentation should provide evidence for a socially relevant fact that has been, owing to 
further social facts, hidden from public sight. Alternatively, it's one of the actual social functions of gender presentation to relay this information. The latter is the best explanation.

The following argument provides greater detail to that explanation. Certain expressions are restricted according to social appropriateness where analogous words serve as euphemistic replacements. For example, fudge stands in for fuck. Notably, transphobic discourse frequently traffics in euphemisms. One example is the question "Have you had the surgery?" This question is often a way of asking, "Do you have a penis or a vagina?" Since it's socially inappropriate to pose such a question to a complete stranger (or even to someone you know, in most circumstances), a "coded" form of discourse is used.

The euphemism involving the terms man/woman and male/female offers interesting variants. The question "Are you a man or a woman?" is often used to euphemistically ask about a person's genitals. We find this kind of euphemism in transphobic representations of a trans person, when people say a person is "really a so and so, disguised as a such and such." Consider the phrases "turned out to be really a man," "living as a woman, although biologically male," and "discovered to be anatomically male." Although most expressions like this needn't be used as euphemistic claims about genitalia, they frequently are. Moreover, if gender presentation literally communicates information about genitalia, in Eurocentered culture it must do so in a euphemistic way, since outright discussion of genitalia is usually restricted. That helps explain how gender presentation could serve this communicative function without being obvious. It also elucidates my claim that gender presentation literally represents genital status: gender presentation euphemistically replaces naked declarations about genital status.

In my view, however, gender presentation isn't merely a euphemism for restricted discourse about genitalia, it's a euphemistic stand-in for genitals per se. As I argued earlier, nakedness is constituted as a distinctive mode of presentation in Eurocentered culture: we can say, crudely, that two modes of self-presentation (naked/clothed) exist. Naked-presentation, like clothedpresentation, is fundamentally informational since it's the locus of a socially salient distinction that is, nonetheless, hidden. Since this display of the information is restricted, however, clothed-presentation serves as its euphemistic replacement (just as it replaces naked declarations about genital status). Moreover, since naked-and clothed-presentation are the two primary, interrelated sensory modes of informational self-presentation, the one is more properly a replacement of the other (rather than, say, the various discursive locutions about genital status).

As the euphemistic replacement of naked-presentation, clothed-presentation effectively references that which it replaces as darn points to damn: a referential structure is instituted whereby female and male modes of nakedness 
are replaced by female and male modes of clothed presentation respectively. Although naked-and clothed-presentations are on the same footing as social modes of self-presentation, the former assumes the fundamentality of referent. Because the referential structure is constituted through a contrast between euphemistic and dysphemistic display, a gap exists between signifier and signified - a barrier between private and public presentations. In this way, the referential structure and the restrictions/allowances on self-presentation are coconstituting within this system of intimacy: it's through being the referent of clothed-presentation that genitalia become morally private.

This account can be used to illuminate the central thesis of the natural attitude: natural sex exists independently of social interactions. As the referent of clothed-presentation, the sexed body appears to be independent of social interactions, so display of the naked body becomes display of the thing itself or the hidden truth. ${ }^{14}$ While naked-presentation is informational, it's a raw display of the bodily truth or reality. What gets obscured is that it's a distinctive, morally infused and regulated modality of self-presentation, rather than some pre-cultural state. ${ }^{15}$ By contrast, clothed-presentation seems secondary and cultural. It becomes a mere appearance that projects a hidden reality: although the naked body isn't available to sight, it's there, somehow, entirely naked. However, one doesn't see the naked body (since the body isn't naked); rather clothed-presentation merely suggests a certain possibility of a naked-presentation. That is, the presence of the body as naked is an illusion created by the referential structure. The naked body is hidden yet suggested in clothed-presentation as the stronger damn is hidden yet suggested in darn. What is truly hidden, once again, is the socially constituted status of naked-presentation as moral phenomenon.

Clearly, the transphobic representation of a trans person as "really an $\mathrm{x}$, disguised as a y" derives from the perceived incongruence between (clothed) gender presentation qua appearance and the sexed body qua reality: because the relation is representational, trans people are read as "deceivers" who falsely project the wrong (naked) body. Moreover, because genitalia are systematically and euphemistically communicated on a regular basis, it's no surprise trans people are frequently subject to forced genital verification to determine real sex (where that status is fundamentally moral in nature): when the euphemistic display of genital information has failed, naked-presentation (or, rather, forced exposure) gives the final, brutal, verdict about the gendered truth.

\section{The Natural Attitude Revisited}

In my account, the natural attitude is not merely a set of beliefs that are taken as axiomatic. Rather, it reflects the way that intimate personhood and the intimate body are actually constituted in Eurocentered culture. It is therefore far 
more than a pre-theoretical "common-sense" about sex; and to change it involves more than a mere change in belief. In this view, genitalia are viewed as essential to sex determination in the natural attitude because they're distinct from all other features with regard to nakedness: they're the completion of the naked body and the locus of moral boundaries between people. Other features such as karyotype don't fall within the purview of nakedness, and therefore are not subject to the same boundaries. Breasts do fall within the purview, but don't possess the same fundamental status. Moreover, genitalia as definitive with regard to sex-differentiated moral structures are the symbolic referents of gender presentation. As the symbolic referents, genitalia are unique as that which is communicated through gender presentation. They constitute the hidden truth of gender. As the hidden truth and the moral completion of nakedness, genitalia are the locus of a metaphysical/moral blending.

It's also clear why the natural attitude expects people to be exclusively male or female. Penises and vaginas complete differential moral structures of the intimate body. The structures of intimate males and females themselves are contrasting and reciprocal. This requires sharply contrasting clothed gender presentation. Indeed, one can argue that the very referential system through which the intimate boundaries are constituted requires a binary: without the possibility of misrepresentation, there could be no possibility of correct representation.

Similarly, it's clear why this distinction should apply exhaustively to people. Since the boundaries that morally differentiate people are fundamentally dichotomized, it becomes impossible for somebody to count as an intimate person without belonging to a sex. Again, insofar as all presentation is genderdichotomized, it becomes impossible for a person to present without being assessed as gender-presenting.

The account explains why only "ceremonial transfers" from one sex to the other are countenanced. Initially perplexing is how something like a masquerade can constitute a ceremonial transfer with regard to genital status. The answer is that gender presentation is a sign of genitalia -it refers to genitalia qua moral completion of nakedness. Indeed, gender presentation as genital representation is involved in the very constitution of nakedness as the hidden truth.

In this view, the social acceptance of ceremonial transfers is explained through the thesis that representation cannot exist without the possibility of misrepresentation. Ceremonial transfers are the social acknowledgment of the possibility of misrepresentation. As such, these occasions do serious cultural work in inscribing gender presentation as representational. Unregulated and widespread misrepresentation would threaten to undermine the very correlation between gender presentation and genital status, and hence its representational function. Therefore transfers need to be recognized while being locally contained. It's little surprise, therefore, that accusations of deception 
(along with forced genital exposure) are used not merely to "expose the truth" but to literally reinscribe the representational nature of gender presentation and thereby reaffirm a particular system of intimate boundaries.

Finally, this account helps explain the thesis that sex is invariant. According to Garfinkel, normals are typically interested in the actual genitalia that "nature" has provided. However, in tough cases they appeal to genitalia to which one is entitled. We can see how this works: Consider a (non-trans) man who has lost his penis in an accident. He will still seem to be intimately male (that is, his body is taken to have a male moral structure). This determination of a male moral structure can easily generate the claim that he ought to have a penis since that body part is the moral completion of the particular moral structure that constitutes his intimate personhood. The structure itself is taken as invariant because it determines his very personhood. Genitalia, although not physically invariant, are "structurally invariant" insofar as they "morally complete" a particular moral structure.

This explains why a trans person who undergoes genital reconstruction surgery may continue to be viewed as "really an $\mathrm{x}$, pretending to be a y." In another essay, I argue that a "misalignment" still exists between gender presentation and genitalia. In such cases, the invariance of sex and the essentiality of genitalia are secured by the ad hoc stipulation that sex is determined by "birth genitalia" and by the representation of the vagina as "artificial" (Bettcher 2007, 49). To this I will now add: The trans woman's body is taken as intimately male. As such her vagina is seen as illegitimate, in part because it's not the completion of the moral structure of her body. In this case, the trans woman has not only "misrepresented" the structure of her body, she has "misrepresented" the genitalia to which she's entitled and which is the moral completion of that structure.

Notably, when a trans woman is assigned an intimate male structure and her actual body looks female, serious tensions arise. What moral status do her breasts and vagina have, if she's understood to be intimately male? Viewing her as male when it comes to her as a viewing subject presents no difficulty. Because she's read as intimately male, she'll be taken to possess the capacity to pre-consensually violate a woman's privacy, and consequently her presence in the women's restroom will raise moral alarm. However, the moral status of breasts and vagina is less clear. This incongruity between body part and perceived boundary may be what motivates the view that she's "sick" or "monstrous." It may also mean her breasts and vagina will not be subject to female boundaries (especially in private). This would mean touching her breasts inappropriately would not count as a female boundary violation in the natural attitude. It would also mean she could not, technically speaking, ever be raped. ${ }^{16}$ Of course, from the perspective of the woman herself, such boundaries would be in place. However, to a "normal" that might mean very little. 
This also helps explain the historically extreme transphobic attitudes of some (non-trans) feminists. For although there may be room for political discussion about women's/womyn's space, for example, Janice Raymond's hurtful claim that all transsexual lesbians effectively rape women by entering their space is beyond the pale (Raymond 1979, 104). The allegation is grounded in the view that transsexual lesbians are "really men" (that is, intimately male) and therefore capable of violating intimate females by intruding upon their privacy. Consider Raymond's remark:

Transsexuals merely cut off the most obvious means of invading women so that they seem noninvasive. However, as Mary Daly has remarked, in the case of the transexually constructed lesbian-feminists their whole presence becomes a "member" invading women's space.... (104)

The accusation is not so different from fairly standard transphobic concerns about women's privacy (for example, restroom use). Moreover, when Raymond claims that "all transsexuals rape women's bodies by reducing the real female form to artifact, appropriating this body for themselves" (104), it may be that the incongruity of a perceived male moral structure with a materially female body is read by her as a kind of monstrous rape. If so, Raymond's feminist view is ironically dependent upon the highly (hetero)sexist system of intimacy that lies at the basis of the natural attitude about sex.

\section{THINKING Resistance}

Given that specific interpersonal boundaries are conventional, in a sense intimate personhood must be viewed as socially conferred. Indeed, one can see how intimate personhood might not be conferred at all to some human beings (in, say, the case of colonial and racist deployments of power). For example, Eurocentered racist representations of some humans as "primitive" or even "animal" can serve the function of denying that such bodies are morally private as a way to justify abusive practices. ${ }^{17}$ Insofar as intimate personhood is essentially gendered in this system, such a denial would place racialized people outside the bounds of the gendered moral order. It would also yield the odd consequence that humans beyond such boundaries cannot be violated, and perhaps can't be intimate persons at all. This consequence is perverse. So it questions the adequacy of my account, since this is an apparent consequence.

One response to this concern is that those who are oppressed tend to inhabit a plurality of cultural "worlds" (see Lugones 1987). This means that while a human may be constituted as one kind of intimate person in one "world" (or perhaps not a person at all), she may be constituted as different kind of intimate person in 
another. Thus, while a trans woman may be constituted as intimately male (and hence invulnerable to violations of the intimate female) in the mainstream "world," she may be constituted otherwise in a trans-friendlier, subaltern "world."

Another answer is that the oppressive "world" itself can deploy contradictory representations of those it oppresses. ${ }^{18}$ For example, it is more accurate to say racist and colonial ideology established liminal change or ambiguous boundaries, rather than denying intimate personhood outright, to racialized people, so that the practitioners of this ideology could genuinely abuse while denying the abuse. This means racialized people were partially and therefore differently situated within the gendered moral order. Similarly, while incarcerated trans women who "have not had the surgery" may be sent to a men's prison (because they are "really men"), they're then especially vulnerable to "rape" (or whatever we decide to call it) while in prison because they're trans women. ${ }^{19}$ The reality, as many trans women know, is that we're both un-rapable (because "intimately male") and entirely rapable (because "ceremonially female").

At any rate, trans people are partially outside of a particular Eurocentered system of sex-differentiated intimacy. Trans resistance can be understood, in part, as a contestation of the boundaries that constitute intimate personhood. Consider that trans bodily dysphoria often concerns morally private parts, which play a role in sex-differentiated structures of the intimate body. Insofar as these parts are loci for interpersonal boundaries delimiting intimate personhood, the phenomenology of the (sexed) body is necessarily a phenomenology of the (gendered) person. In part, then, bodily dysphoria can involve unhappiness with the socially conferred interpersonal boundaries and a desire to renegotiate them.

Furthermore, consider gender presentation. In my account, although it doesn't literally modify the body, it has the capacity to symbolically confer a sexed body that is "visibly" present "under one's clothes." Given the role of the intimate body in the boundaries that delimit intimate personhood, gender presentation can be understood not only as a technology of the body, but as a technology of intimate personhood. That is, it can be used as a way to redraw one's interpersonal boundaries. Indeed, it is possible to undermine the very capacity of gender presentation to communicate genital status at all. This is already part of the actual gender practice in certain trans-friendly, subaltern contexts, and it seems to reflect how some trans people literally "opt out" of the mandatory system of genital disclosure (Bettcher 2009). This clearly involves a radical reconfiguration of interpersonal boundaries and therefore intimate personhood itself. It's what's required, I believe, when one is at odds with the moral and metaphysical order itself: one needs—we need —new kinds of self, new modalities of intimacy, in order to exist. 


\section{Notes}

I wish to thank Kathryn Hill for her invaluable work as my research assistant. The combination of her effort and talent significantly enriched this essay (and other research that I hope will follow). As always, I express deep gratitude to Susan Forrest for her insights and loving provocations. This essay is part of a larger project that focuses on the nature of transphobia and transphobic violence. I have presented some of this material in nonphilosophical forums. The site learningtrans.org has a link to some related videos of performance pieces and presentations that explore these issues in depth (http:// learningtrans.org/contributors/bettcher-talia/full-frontal/).

1. For more details, see Bettcher 2007.

2. They're also bound up with notions of dignity and shame, but that's beyond this current discussion.

3. Although my account has implications for the right to privacy, my goal is neither to provide an account nor to engage with the literature on that topic. My discussion is limited mostly to privacy over bodily access (as opposed to privacy over informational access or decisional privacy).

4. See Nagel 2002 for an interesting discussion.

5. In this paper, I remain neutral on whether person applies cross-culturally/historically. To be sure, it's important to avoid ethnocentric assumptions about what a person is and whether any one single notion applies universally. The other danger, however, is to restrict personhood to a culture and then deny personhood to other humans (see my p. 12-3 above). In my use of person, the empirical question is whether boundarygrounded intimacy is trans-cultural. It's enough for my argument to note that many cultures possess relational, bounded intimacy as a social possibility and that the boundary systems in these cultures differ significantly.

6. I characterize intimacy independently of love. Certainly intimacy and love are closely associated (largely because people equate intimacy with emotional intimacy). However, we must also square with physical intimacy. Moreover, emotional intimacy needn't involve love. Consider the possibility of intimate partner violence that presupposes an intimacy, while rendering problematic the application of the word "love." The latter may well be used by an abuser ("I hit you because I love you"), but its applicability is highly doubtful.

7. This is hardly the first attempt to understand the value of privacy in terms of intimacy. This may be the first account to see moral privacy as constitutively required from intimacy (where intimacy is understood in terms of interpersonal "proximity"). For other accounts see Fried 1970; Rachels 1975; Gerstein 1978; Inness 1992.

8. This can integrate accounts that find the value of privacy in intimacy and accounts that find it in the person herself. For an account of the latter type, see Reiman 1976.

9. Although I cannot defend it fully here, I see these boundaries as more basic than a right to privacy (which might be explained in terms of them).

10. Delicate questions arise about whether boundaries on the female face help constitute female nakedness as such or whether these boundaries operate orthogonally. Related questions arise regarding cultural practices of gendered facial decoration. For 
example, in Eurocentered culture, women sometimes claim to "feel naked" in public without makeup. Such considerations raise fascinating questions that I cannot address in this paper. I'm grateful to an anonymous referee for raising some of these issues.

11. It is important to distinguish between nakedness (as a specific social and moral construct) and more general negotiations of intimacy. Although exposure of genitals may "complete" nakedness as a social construct, this doesn't mean a given person will experience such exposure as maximally physically intimate.

12. See the California Penal Code, Part One, Title 9, Ch. 1, § 261a in Deering (2010).

13. This doesn't mean a male cannot have his bodily privacy violated by a female. Nor does it mean a female cannot violate a male through a decency offense (although this seems unlikely). Such violations concern the non-consensual violations of boundaries, whereas my point concerns pre-consensual violations. The former is "shaded" by the asymmetry in the latter.

14. For various ways in which the naked body has been represented as a truth, see Barcan 2004, 97-106.

15. In my view, even sex is socially constructed. I also insist, however, that sex and gender are constructed as distinct from each other. A full discussion of this important issue is well beyond the scope of this paper.

16. That said, because she looks sufficiently female, it may be expected that she abide by certain boundaries in public.

17. The case of Saartjie Baartman is one obvious example. For extended discussions of the role of nakedness in colonial and racist ideology, see Barcan 2004, 150-65.

18. For an excellent discussion of this idea, see Scheman 1996.

19. According to a recent study, transgender inmates in California prisons reported sexual victimization over a lifetime at the stunning prevalence of $75 \%$. See Sexton, Jenness, and Sumner 2009, 24.

\section{REFERENCES}

Barcan, Ruth. 2004. Nudity: a cultural anatomy (Dress, body, culture). New York: Berg Publishers.

Bettcher, Talia Mae. 2007. Evil deceivers and make-believers: Transphobic violence and the politics of illusion. Hypatia 22 (3): 43-65.

. 2009. Trans identities and first-person authority. In You've changed: sex reassignment and personal identity, ed. Laurie Shrage. Oxford: Oxford University Press.

Bornstein, Kate. 1994. Gender outlaw: on men and women and the rest of us. New York: Routledge.

Deering's California Codes Annotated. 2010. Matthew Bender \& Company, Inc., LexisNexis Group.

Fried, Charles. 1970. An anatomy of values. Cambridge: Harvard University Press.

Garfinkel, Harold. 1967. Studies in ethnomethodology. Englewood Cliffs, N.J.: PrenticeHall.

Gerstein, Robert S. 1978. Intimacy and privacy. Ethics 89 (1): 76-81. 
Hale, C. Jacob. 1996. Are lesbians women? Hypatia 11 (2): 94-121.

- 1997. Leatherdyke boys and their daddies: How to have sex without women or men. Social Text (52/53): 223-36.

- 1998a. Consuming the living, dis(re)membering the dead in the butch/FTM borderlands. GLQ: A Journal of Lesbian and Gay Studies 4 (2): 311-48.

- 1998b. Tracing a ghostly memory in my throat. In Men doing feminism, ed. T. Digby. New York: Routledge.

Harger, Jim. 2010. Group wants driver's licenses for transgender people to be an issue in Michigan Secretary of State race. Grand Rapids Press, January 30.

Heywood, Todd E. 2010. Paul Scott targets transgendered people in race for Secretary of State. Michigan Messenger, January 21.

Husserl, Edmund. 1983. Ideas pertaining to a pure phenomenology and to a phenomenological philosophy, First Book: General Introduction to a Pure Phenomenology, trans. K. Schuhmann. Dordrecht: Kluwer Academic Publishers.

Inness, Julie C. 1992. Privacy, intimacy, and isolation. New York: Oxford University Press.

Kessler, Suzanne J., and W. McKenna. 1978. Gender: an ethnomethodological approach. New York: John Wiley and Sons.

Lugones, María. 1987. Playfulness, "world"-traveling, and loving perception. Hypatia 2 (2): $3-19$.

Nagel, Thomas. 2002. Concealment and exposure. In Concealment and exposure and other essays. New York: Oxford University Press.

Rachels, James. 1975. Why privacy is important. Philosophy and Public Affairs 4 (4): 323-33.

Raymond, Janice. 1979. The transsexual empire: the making of the she-male. Boston: Beacon Press.

Reiman, Jeffrey. 1976. Privacy, intimacy, and personhood. Philosophy and Public Affairs 6 (1): 26-44.

Scheman, Naomi. 1996. Queering the center by centering the queer: Reflections on transsexual and secular Jews. In Feminists rethink the self, ed. Diana Tietjens Meyers. Boulder, CO: Westview Press.

Sexton, Lori, Valerie Jenness, and Jennifer Macy Sumner. 2009. Where the margins meet: A demographic assessment of transgender inmates in men's prison. Justice Quarterly 1 (32): 1-32.

Watson, Irene. 1998. Naked peoples: Rules and regulations. Law/Text/Culture 4 (1): $1-17$. 